\title{
A rare endoscopic appearance of primary gastrointestinal mantle cell lymphoma resembling ulcerative colitis
}

\author{
Koksal AS ${ }^{1}$, Taskiran $\mathrm{I}^{1}$, Kalkan $\mathrm{IH}^{2}$, Kayacetin $\mathrm{E}^{1}$ \\ Türkiye Yüksek Ihtisas Hospital, Department of Gastroenterology, Ankara, Turkey. koksalas@yahoo.com
}

To the Editor,

Mantle-cell lymphomas (MCL) account for 1-8\% of non-Hodgkin's lymphomas and typically occur in male patients older than 55 years of age. Patients often present at an advanced stage of the disease with widespread involvement of the extranodal sites. Macroscopic involvement of the gastrointestinal (GI) tract occurs in 10-28 \% of the cases (1). Microscopic involvement is much higher and occurs in up to $92 \%$ of cases (2). Patients present with abdominal pain, diarrhea, hematochezia, weight loss and fatigue. Herein we present a case of primary GI MCL with an unusual form of endoscopic presentation in whom the symptoms and endoscopic appearance of the colon initially suggested ulcerative colitis.

A 73-year-old male patient was admitted for weight loss and watery mucoid diarrhea in the last two months. Physical examination was unremarkable. Laboratory findings revealed a chronic anemia (Hb: $9.5 \mathrm{~g} / \mathrm{dl})$ with elevated erythrocyte sedimentation rate $(74 \mathrm{~mm} / \mathrm{h})$ and CRP $(10.1 \mathrm{mg} / \mathrm{dl})$ levels. Biochemical findings including the liver function tests, renal tests and LDH were all within normal limits. Colonoscopy of the left colon revealed diffuse mucosal fragility, edema, erosions, linear ulcerations and scattered polyps covered with white exudates (Fig. 1). The proximal colon segments had a distorted vascular network and slight mucosal granularity. Endoscopic findings were compatible with the diagnosis of ulcerative colitis. But the histopathological examination of the biopsy specimens revealed atypical lymphoid proliferation consistent with MALT lymphoma. Upper endoscopy showed hyperemia, edema and small nodular lesions throughout the fundus, corpus and antrum. Gastric biopsies also suggested a gastric MALT lymphoma but immunohistochemical profile of the gastric and colonic specimens corresponded to a MCL (CD5(+), CD20(+), CD23(-), cyclin D1(+), ki-67: $20 \%$ ). Helicobacter pylori was negative. Positron emission tomography

${ }^{1}$ Türkiye Yüksek ihtisas Hospital, Department of Gastroenterology, Ankara, and ${ }^{2}$ Kirikkale University Faculty of Medicine, Department of Gastroenterology, Kirikkale, Turkey

Address for correspondence: AS Koksal, 1443. cadde, 40/13 Çukurambar, Ankara, Turkey.

Phone: +90.312.3061334, Fax: +90.312.3124120

Text in PDF www.elis.sk.

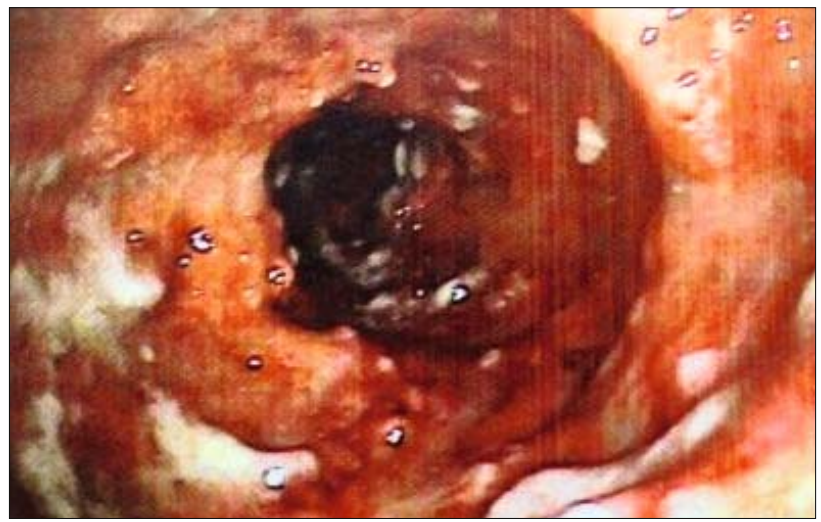

Fig. 1. Colonoscopic examination revealed diffuse mucosal fragility, edema, erosions, linear ulcerations and scattered polyps covered with white exudates resembling ulcerative colitis.

showed diffuse involvement of the stomach, descending, sigmoid colon and rectum. There was no bone marrow involvement. The patient was transferred to the Oncology Department where he started to receive standard R-CHOP (rituximab, cyclophosphamide, adriamisin, vincristine, prednisone) chemotherapy.

The typical endoscopic appearance of early MCL is numerous small nodular or polypoid tumors termed multiple lymphomatous polyposis (MLP). On the other hand, advanced MCL presents in three different forms: ulcerated, diffuse infiltrating, and elevated. It is frequently associated with involvement of extra-intestinal sites such as intraabdominal lymph nodes and/or bone marrow. Romaguera reported the colonoscopic findings of 60 patients with MCL as follows: normal in 31, nodules in 14, polyps in 12, inflammation, ulcers, and thickness in 1 patient, each (3).

Our patient had diffuse mucosal fragility, erosions, linear ulcerations and polyps in the left colon. The symptoms and endoscopic appearance of the colon was initially suggestive for ulcerative colitis. But the final diagnosis was primary GI MCL. Primary GI MCL constitutes only $2 \%$ of the patients with primary GI lymphoma and presents in the form of MLP (4). Non-MLP form of primary MCL is very rare. Tamura et al. reported a case of primary MCL in whom colonoscopy showed diffuse redness with erosion continuing from rectum to ileum and simulating ulcerative colitis (5). Our case was unique because it demonstrated a rare case of pri- 
mary MCL having predominantly inflammatory features. Diffuse mucosal fragility, erosions and linear ulcerations were in favor of ulcerative colitis. Small polyps covered with white exudates were initially thought to be pseudopolyps. But review of the endoscopic pictures after the histologic diagnosis of MCL revealed that they were lymphamotous polyps in fact.

In conclusion, primary GI MCL may not only present as MLP but also predominantly in the form of colitis which may resemble ulcerative colitis.

\section{References}

1. Zucca E, Stein H, Coiffer B. European lymphoma task force (TLTF): report of the workshop on mantle cell lymphoma (MCL). Ann Oncol 1994; 5: 507-511.
2. Salar A, Juanpere N, Bellosillo B, Domenech E, Espinet B, Seoane $\mathrm{S}$ et al. Gastrointestinal involvement in mantle cell lymphoma: a prospective clinic, endoscopic, and pathologic study. Am J Surg Pathol 2006; 30: 1274-1780.

3. Romaguera JE, Medeiros LJ, Hagemeister FB, Fayad LE, Rodriguez MA, Pro B et al. Frequency of gastrointestinal involvement and its clinical significance in mantle cell lymphoma. Cancer 2003; 97: 586-791.

4. Morton JE, Leyland MJ, Vaughan Hudson G, Vaughon Hudson B, Anderson L, Bennett MH et al. Primary gastrointestinal non-Hodgkin's lymphoma: a review of 175 British National Lymphoma Investigation cases. Br J Cancer 1993; 67: 776-782.

5. Tamura S, Ohkawauchi K, Yokoyamam Y, Higashidani Y, Daibata $\mathbf{M}$, Hiroi $\mathbf{M}$ et al. Non-multiple lymphomatous polyposis form of mantle cell lymphoma in the gastrointestinal tract. J Gastroenterol 2004; 39: 995-1000. 